\title{
The emerging role of HHV-6 in neurologic disease: lesson from multiple sclerosis and epilepsy Steven Jacobson*
}

Address: Chief, Viral Immunology Section, National Institute of Neurological Disorders and Stroke, National Institutes of Health, Building 10, Room 5B-16, Bethesda, Maryland, 20892, USA

* Corresponding author

from 2006 International Meeting of The Institute of Human Virology

Baltimore, USA. 17-2I November, 2006

Published: 2I December 2006

Retrovirology 2006, 3(Suppl I):S64 doi:10.1 I86/I742-4690-3-SI-S64

(C) 2006 Jacobson; licensee BioMed Central Ltd.

Human herpesviruses 6A and 6B (HHV-6A and HHV-6B) are lymphotropic herpes viruses that play a role in the pathogenesis of roseola and febrile illness. HHV-6 is considered a common, ubiquitous virus of humans with a seroprevalence of greater than $90 \%$ in the general population. The HHV6 variants have been shown to be distinct and have different cell tropisms, disease associations and reactivity to monoclonal antibodies. While HHV-6B is spread through saliva, the mode of transmission of HHV$6 \mathrm{~A}$ is not known. HHV-6 predominantly infects CD4 cells, adversely affecting function. HHV-6A, but not HHV-6B also invades CD8 cells. HHV-6 is increasingly being recognized as an emerging pathogen with recently reported associations of both CNS and systemic disease. These include disorders associated with seizures and encephalitis, transplant morbidity, multiple sclerosis, epilepsy, cancer, drug induced hypersensitivity syndrome, and immunosuppression. As HHV-6 has been linked to a number of chronic, human disorders further studies are required to elucidate the causal relationship of this virus to the pathogenesis of such diseases. Since our first detection of HHV-6 DNA in diseased brain tissue from a subset of epilepsy patients, an extended cohort of patients with or without mesial temporal sclerosis has been progressively studied, confirming our preliminary results on an association of HHV-6 CNS infection and mesial temporal lobe epilepsy. To validate the extent and distribution of HHV-6 in glial cells, brain tissue from surgical resections was processed to establish cultures of primary astrocytes. The detection of viral late gene transcripts confirmed the presence of active infection in these cultured cells. Viral
DNA was documented in cultured astrocytes by quantitative TaqMan and nested PCR. This is the first demonstration of an HHV-6 infection detected in vivo and maintained in primary cultured astrocytes. These results confirm our previous findings on epilepsy brain resections and support an association of HHV-6 active infection in this disorder. These data open a new perspective into the study of pathogenesis of mesial temporal lobe epilepsy. 